\title{
CONCEPCIONES DE FUTUROS PROFESORES DEL PRIMER CICLO DE PRIMARIA SOBRE LA NATURALEZA DE LA CIENCIA: CONTRIBUCIONES DE LA FORMACIÓN INICIAL
}

THOMAZ, M.F., CRUZ, M.N., MARTINS, I.P. y CACHAPUZ, A.F. Universidade de Aveiro. 3810 Aveiro. Portugal.

\section{SUMMARY}

Research studies have showen that the image of science that students construct during their formal learning is strongly influenced by the teacher's conceptions about the nature of scientific knowledge. It is then fundamental to investigate the impact of initial teacher's education in the development of those conceptions in future teachers.

The aim of this article is to present a study about the contribution of the three year initial teacher education, of basic education teachers ( 1 st cycle - 6/10 years olds), in the development of their conceptions about the nature of science. The results show that the initial teacher education, as it has been developed in teacher training institutions, does not significantly influence the ideas held by future teachers about the nature of science.

\section{INTRODUCCIÓN}

Un aspecto sobresaliente de aigunos proyectos de desarrollo curricular en las décadas de los setenta y ochenta es el énfasis dado a la importancia de la construcción, desde los primeros años, de representaciones sobre la ciencia y el conocimiento científico. En muchos de tales proyectos se declara como objetivo la comprensión de la naturaleza de la ciencia por parte de los alumnos. Sin embargo algunos resultados (Anderson et al., 1986; Yager, 1984; Lerner y Bennetta, 1988) han mostrado que, tras varios años de enseñanza formal, los alumnos no parecen comprender aspectos relativos a cómo se produce el conocimiento científico. La misma conclusión es aplicable a los futuros profesores de ciencias (Ogunniyi, 1982; Bloom, 1989; Aguirre et al., 1990). Una inferencia que se ha venido haciendo es que Ia enseñanza de ciencias en cualquier nivet nunca ha contemplado la dimensión epistemológica debido a lagunas en la formación inicial y continua de los profesores (Lakin y Wellington, 1994).
Esta afirmación presupone que los profesores deben conocer aspectos relevantes de la naturaleza de la ciencia y saber como transmitirla a sus alumnos (Lantz y Kass, 1987; Duschl y Wright, 1989). Una formación adecuada de los profesores de ciencias deberá contemplar, además del saber científico, conocimientos sobre las finalidades de la ciencia, sus métodos, sus relaciones con la sociedad y la tecnología, la naturaleza del conocimiento científico y su construcción. La cuestión es doblemente importante, ya que desde una perspectiva constructivista del proceso de enseñanza-aprendizaje, defendida actualmente por un número cada vez mayor de educadores (Pope y Gilbert, 1983; Driver y Bell, 1985; Driver y Oldham, 1986; Johnstone, 1987; Forman, 1992; Gil, 1992, Ritchie y Cook, 1994; Shepardson y Moje, 1994), una visión particular sobre la naturaleza de la ciencia influye no sólo en lo que se enseña sino en cómo se enseña ciencias (Salmon, 1988; Lakin y Wellington, 1994). 
Estamos convencidos de que la enseñanza de ciencia y tecnología en la educación primaria puede contribuir a que los niños desarrollen una actitud positiva y meditada respecto a la ciencia, lo que les podrá ayudar a una mejor insercion en la sociedad actual y futura, caracterizada por una fuerte componente científico-técnica (Harlen, 1987). Los alumnos construyen desde muy temprano representaciones sobre la ciencia que no son fáciles de modificar. De ahí la importancia dada a la calidad de la contribución de la formación inicial de los profesores para la construcción de imágenes positivas sobre lo que es la ciencia.

Actualmente, a pesar de que ya haya estudios que evidencian el predominio de puntos de vista empiristas en los profesores portugueses (Cachapuz y Praia, 1992), no se conocen estudios sobre las concepciones que estos futuros profesores poseen acerca de tales aspectos cuando son alumnos.

El proyecto CInEB desarrollado en la Universidad de Aveiro (Portugal) pretende, entre otras cosas, contribuir a la identificación de obstáculos para la innovación en enseñanza de la ciencia y la tecnología, lo que permitirá fundamentar propuestas para mejorar la formación de profesores. En este marco se admite que las representaciones de los futuros profesores sobre la naturaleza de la ciencia y su enseñanza-aprendizaje pueden llegar a incluirse entre tales obstáculos.

Es de esperar que las concepciones dominantes entre los alumnos, sobre la naturaleza del conocimiento científico, al iniciar cursos específicos de formación del profesorado, no sean muy diferentes de las dominantes entre los profesores que les enseñaron en años precedentes. Es, pues, necesario que la formación inicial contribuya al cambio conceptual hacia puntos de vista racionalistas y constructivistas defendidos por los filósofos de la ciencia contemporáneos. Estos dos aspectos constituyen nuestra hipótesis de trabajo.

Así, el objetivo principal del estudio que se presenta es investigar:

a) las concepciones de los futuros profesores del Ir. ciclo de enseñanza primaria ( 6 a 10 años) respecto a la naturaleza de la ciencia -sus objetivos, procesos seguidos por los científicos en su construcción, naturaleza del conocimiento científico y su papel en la sociedati-;y

b) cuál es la evolución de tales concepciones durante la formación inicial.

\section{MÉTODO}

Aunque este no sea un estudio longitudinal, creemos que el modelo de trabajo adoptado (por comparación de muestras) es adecuado metodológicamente, ya que cualquiera de las muestras de alumnos de $1^{\circ}$ y $3^{\circ}$ encuestados son características de tales cursos.
Los datos se han obtenido mediante la utilización en dos fases de un test escrito constituido por preguntas abiertas. Fn la primera fase se pasó a 90 futuros profesores de 1r. ciclo de enscñanza primaria, Ios cuales cursaban en 1990-91 su tercer año (último curso de la formación inicial), estando ya en período de prácticas. los centros que en Portugal dan cuenta de la formación del profesorado son: CIFOP (Centros Integrados de Formación de Profesores, integrados en las universidades) y ESE (Escuelas Superiores de Educación, integradas en institutos politécnicos). De los trece centros existentes sólo se seleccionaron cinco, que se consideraron representativos de los diferentes modelos de formación inicial. En cada uno de ellos se escogió aleatoriamente uno de los grupos para ser estudiado.

En la segunda fase se pasó, al comienzo del curso 1991 92 , el mismo cuestionario a 70 alumnos que comenzaban su primer año de formación inicial como profesores de 1r. ciclo.

Se hizo en el curso 1990-9I un estudio piloto con un grupo de alumnos de $1^{\circ}$. El cuestionario formado por 16 preguntas abiertas trataba de identificar concepciones sobre la naturaleza de la ciencia, sobre la enseñanza de la ciencia y sobre el modo en que se entiende que los niños aprenden ciencias.

En este artículo sólo se analizan las respuestas a cuestiones relacionadas con la naturaleza de la ciencia (fines, procesos de construcción, naturaleza del conocimiento científico, estatus epistemológico de las teorías y leyes científicas, y relación ciencia-sociedad).

El cuestionario fue validado por cinco investigadores en didáctica de la ciencia. Se administró en cada uno de los centros seleccionados como encuesta voluntaria, sin límite de tiempo. Las respuestas fueron anónimas e individuales.

\section{RESULTADOS Y DISCUSIÓN}

En esta sección se presentan los resultados obtenidos del análisis de las respuestas a las cuestiones relacionadas con cinco aspectos de la naturaleza de Ia ciencia:

a) fines de la ciencia;

b) procesos seguidos por los científicos en la construcción de la ciencia;

c) naturaleza del conocimiento científico;

d) estatus epistemológico de las teorías y leyes científicas;

e) relacion ciencia-sociedad.

El método adoptado para el análísis del contenido siguió el modelo de Erickson (1979) para la construcción de categorias de respuesta: por comparación entre las propiedades definitorias de las respuestas y las previstas para la respuesia adecuada. Las categorías de respuesta, por tanto, no están predeterminadas. Pertenecen a la 
misma categoría las respuestas que reflejan ideas equivalentes.

\section{FINES DE LA CIENCIA}

Se ha sostenido que la eđucación científica de los profesores de 1r. ciclo de primaria debería facilitar la reflexión sobre la ciencia (como conocimiento), la actividad científica, la historia y filosofía de la ciencia, y la interacción ciencia-sociedad (DuschI, 1985), de modo que puedan desarrollar en sus alumnos puntos de vista sobre la ciencia que les sean útites como futuros ciudadanos. En efecto, la ciencia tiene por fín el aumento del conocimiento científico en sí mismo y la resolución de los problemas de la humanidad (desde el punto de vista social), del planeta e incluso del universo. Esto puede inchuir la búsqueda-indagación de nuevos métodos e instrumentos. Así, la ciencia puede contribuir a desarroIlar Ia tecnología, beneficiándose a su vez de ésta.

Esta múltiple visión respecto a las finalidades de la ciencia (que la ciencia está hecha por individuos y para individuos, que se empeñan en crear conocimiento cientifico en sí mismo, lo cual ayudará a resolver los problemas existentes y evitará que aparezcan otros) es lo que deseamos que adquieran los alumnos después de su formación científica. Sin embargo, los resultados de este estudio muestran que sólo un porcentaje muy pequeño de los futuros profesores investigados constató tal visión.

En la tabia I se muestra la distribución en categorías de las respuesta dadas por los alumnos de $1^{\circ}$ y $3^{\circ}$ a las cuestiones:

¿Cuf́les son a tu entender los objetivos ofinalidades de la ciencia?

¿Cuál es desde tu punto de vista el objetivo del trabajo de los cientificos?

Tabla I

Concepciones dominantes entre los futuros profesores sobre los fines de la ciencia.

\begin{tabular}{|l|c|c|}
\hline Categoriá de respuesta & $\begin{array}{c}\text { Ir. curso } \\
\%(\mathbf{N}=90)\end{array}$ & $\begin{array}{l}\text { 3r. curso } \\
\%(\mathrm{~N}=70)\end{array}$ \\
\hline $\begin{array}{l}\text { Búsqueda de conocimientos } \\
\text { independientemente de so } \\
\text { aplicación }\end{array}$ & 65,7 & 69,5 \\
\hline $\begin{array}{l}\text { Búsqueda de conocimientos } \\
\text { teniendo en cuenta su } \\
\text { aplicación }\end{array}$ & 25,7 & 25,3 \\
\hline $\begin{array}{l}\text { Búsqueda de conocimientos } \\
\text { teniendo en cuenta los dos } \\
\text { aspectos complementarios }\end{array}$ & 8,6 & 2,1 \\
\hline No contesta & 0,0 & 3,1 \\
\hline
\end{tabular}

El $65,7 \%$ de los encuestados de $1^{\circ}$ y el $69,5 \%$ de los de $3^{\circ}$ muestran ideas acerca de los objetivos de la ciencia desde una posición académica; es decir, desligada de su aplicación y función social.

Ejemplos de las respuestas que establecen que el objetivo de la ciencia es la búsqueda de conocimientos independientemente de su aplicación son:

\section{[...] descubrir y comprender. (lr. año)}

[...] los objetivos o finalidades de la ciencia son cl estudio de los fenómenos, teniendo siempre en cuenta la verdad, para lo que será necesario el máximo rigor para encontrár tal verdad. (3r. año)

El análisis de los resultados obtenidos en los dos grupos de encuestados sobre sus concepciones respecto a la finalidad de la ciencia sugiere que los tres affos de formación especifica de los profesores de 1r. ciclo no influyen en tales concepciones. Esta situación se observa también en los individuos para los que el objetivo de la ciencia es buscar el conocimiento pero teniendo en cuenta su aplicación.

Ejcmplos de respuestas que revelan esta última visión son:

Estudiar todo lo que nos rodea y nos habla de [...] con el fin de resolver problemas prácticos. (3r. año)

[...] la promoción del progreso, del desarrollo, de la cultura, etc., batiéndose y luchando stempre por el bienestar social. (1r. año)

Sólo el $8,6 \%$ de los encuestados de $1^{\circ}$ y el $2,1 \%$ de los de $3^{\circ}$ expresan la idea de que el fin de la ciencia contempla los dos aspectos complementarios, como se puede inferir del siguiente ejemplo transcrito:

A mi modo de ver, la ciencia ayuda no sólo a desarrollar áreas que se relacionan con la vida diaria, o del individuo en la sociedad, sino que también ofrece una visión más amplia y cada vez más específica del mundo que no rodea. (3r. año)

\section{Procesos seguidos por los científicos en la construc- ción de la ciencia}

En el contexto de Ia llamada «Nucva Filosofía de la Ciencia», compartida en lo fundamental por los filósofos de la ciencia contemporáneos, se reconoce que la metodología científica es diversa debido a la complejidad del trabajo científico, a las distintas posiciones filosóficas respecto a la epistemología de la ciencia y a las diferencias existentes entre los científicos tanto respecto a su área de conocimiento como a su personalidad (White, 1983; Giere, 1989; Gilbert, 1991).

Aunque no sea posible describir un único método, esto no significa que la ciencia no tenga métodos. Puesto que la construcción del conocimiento científico es una actividad social compleja, existe un pluralismo metodológico, una gran variedad de procesos disponibles; cada científico adopta los que considera más apropiados para 
el ámbito y estructura del tema a investigar, siendo compartidos por sus colegas.

En la tabla II se presentan las categorias de respuestas de los alumnos de $1^{\circ}$ y $3^{\circ}$ relativas a la cuestión:

¿Cual es tu opinión sobre los procesos seguidos por los científicos en la construcción de la ciencia?

\section{Tabla II}

Concepciones dominantes entre los futuros profesores respecto a los procesos de construcción de la ciencia.

\begin{tabular}{|l|c|c|}
\hline Categoría de respuesta & $\begin{array}{c}\text { 1r. curso } \\
\%(\mathbf{N}=\mathbf{9 0})\end{array}$ & $\begin{array}{c}\text { 3r. curso } \\
\%(\mathbf{N}=\mathbf{7 0})\end{array}$ \\
\hline $\begin{array}{l}\text { Visión empirista/inductivista } \\
\text { de los procesos de construcción } \\
\text { de la ciencia }\end{array}$ & 47,1 & 53,7 \\
\hline $\begin{array}{l}\text { Visión pluralista de los procesos } \\
\text { de construcción de la ciencia }\end{array}$ & 2,9 & 2,1 \\
\hline Otras & 34,3 & 25,3 \\
\hline No contesta & 5,7 & 18,9 \\
\hline No sabe & 10,0 & 0,0 \\
\hline
\end{tabular}

Los resultados de este estudio evidenciaron que el $47,1 \%$ de Ios alumnos de $1^{\circ}$ adoptan una visión empirista respecto a la construcción del conocimiento e ideas inductivistas sobre «el método de la ciencia», enfatizando que la observación es el único punto de partida para el desarrollo del conocimiento. No es de extrañar tal resultado en vista de los obtenidos por Praia y Cachapuz (1993). Según éstos, de una muestra de profesores portugueses de ciencias, cerca del $66,7 \%$ presentaba una postura empirista respecto a la naturaleza de la ciencia. Lo que es más preocupante es que la mayoría de los alumnos de $3^{\circ}(53,7 \%)$ también muestra esta postura, poniendo de manifiesto que la formación inicial en nada contribuyó a este cambio conceptual.

Ejemplos de respuestas de esta categoría son:

[...] usando el método científico [...] observación, hipótesis, experimentación y conclusión. (1r. curso)

Observación, establecimiento de hipótesis, experimentación y discusión đe la experiencia hecha, conclusión y generalización. (3r. curso)

$1^{\circ}$, observación; $2^{\circ}$, hipótesis; $3^{\circ}$, desarrollo; $4^{\circ}$, establecimiento de cuestiones; $5^{\circ}$, conclusión. ( $3 r$. curso)

Es preocupante que sólo un pequeño porcentaje de los investigados revele una visión pluralista de los procesos de construcción de la ciencia $\left(2,9 \%\right.$ y $2,1 \%$ en $I^{\circ}$ y $3^{\circ}$ respectivamente). Es también preocupante la falta de influencia de los tres años de formación como futuros profesores de ciencias respecto a este punto de vista. Un ejemplo de ello es:

A lo largo del tiempo, la ciencia tiende a evolucionar, los científicos recurren a los procesos a su alcance en su época, su estilo y todo lo que los rodea, para construir la ciencia. (3r. curso)

El análisis de las respuestas a esta pregunta muestra que existe un porcentaje relativamente grande de opiniones que no constituyen propiamente una respuesta a la cuestión, imposibles de agrupar en categorías distintas, por lo que han sido incluidas en una única, denominada "otras». Puede que esto sea debido a que los alumnos no hayan entendido la pregunta o a que no poseyeran ideas concretas sobre este aspecto.

Ejemplos de respuestas de este tipo son:

[...] delimitan su área de estudio; sus contenidos, sus objetivos, métodos e investigación. (ir. curso)

[...] poner en práctica aquello que se presenta como teórico, intentando explicar lo que nos todea. (3r. curso)

Creo que, al construir ciencia, los científicos deben comenzar desde el punto más pequeño para poder explicar el mayor o, al contrario, fijarse en el mayor y descubrir su porqué. (3r. curso)

[...] a veces no tienen en cuenta los factores externos, que son la propia realidad. (3r. curso)

[...] son procesos muy complejos y eficientes. (1r. curso)

Es también preocupante que después de la enseñanza específica haya todavía un $18,9 \%$ que no responde a la pregunta.

Se puede deducir que, en lo concerniente a los procesos de construcción del conocimiento científico, la formación de estos futuros profesores ha reforzado una concepción empirista/inductivista y no una concepción pluralista, en la que se admita la existencia de una gran variedad de métodos dependiendo del carácter de la investigación y del propio investigador.

\section{La naturaleza del conocimiento científico}

Según la nueva fílosofía de la ciencia, la construcción de la ciencia es un intento de comprensión del mundo, y el conocimiento cientificico nunca debe ser identificado con la verdad. Este conocimiento tiene un estatus puramente temporal, tal como hace notar Cleminson (1990). Ei nuevo conocimiento es producido por actos creativos de la imaginación ligados a métodos de indagación, siendo su aceptación problemática y frecuentemente poco fácil. Aunque sea incapaz de interpretar nuevas situaciones, el abandono del conocimiento previamente aceptado se produce usualmente con gran resistencia. Ésta es la posición que defendemos y que esperaríamos que fuese compartida por los futuros profesores de ciencia. 
En la tabla III se presentan las frecuencias de las ideas de los alumnos de $1^{\circ}$ y $3^{\circ}$ relativas a la cuestión:

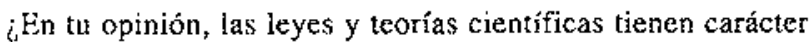
permanente o temporal? Da ejemplos que puedan ilustrar tu respuesta.

Tabia III

Concepciones dominantes entre los futuros profesores respecto a la naturaleza del conocimiento científico

\begin{tabular}{|l|c|c|}
\hline Categoría de respuesta & $\begin{array}{c}\text { 1r. curso } \\
\%(\mathbf{N}=90)\end{array}$ & $\begin{array}{c}\text { 3r. curso } \\
\%(\mathbf{N}=70)\end{array}$ \\
\hline $\begin{array}{l}\text { Visión dinámica del } \\
\text { conocimiento científico }\end{array}$ & 85,7 & 74,7 \\
\hline $\begin{array}{l}\text { Visión estática del } \\
\text { conocimiento científico }\end{array}$ & 11,4 & 17,9 \\
\hline Otras & 2,9 & 3,2 \\
\hline No contesta & 0,0 & 4,2 \\
\hline
\end{tabular}

Se puede deducir de los resultados de este estudio que la visión dinámica de la naturaleza del conocimiento científico es compartida por la gran mayoría de los alumnos tanto de $1^{\circ}(85,7 \%)$ como de $3^{\circ}(74,7 \%)$, aunque los resultados relativos a los alumnos de $1^{\circ}$ sean más positivos.

\section{Un ejemplo típico de esta categoría es:}

En mi opinión, las leyes y teorías científicas son temporales, puesto que aquello que se puede considerar científicamente correcto hoy, puede no serlo mañana [...] (1r. curso)

Sin embargo, persiste un $11,4 \%$ de alumnos de $1^{\circ}$ y un $17,9 \%$ de los de $3^{\circ}$ que presentan una visión estática del conocimiento científico, como se puede ilustrar con las siguientes respuestas:

I as leyes tienen un carácter permanente, pues para llamarse así tienen que haber sido debidamente comprobadas y sobre cllas no restan dudas. Las teorías tienen un carácter temporal, pues pueden y deben ser puestas en duda siempre que surjan nuevos hechos para los que no tengan respuesta. (1r. curso)

Ias leyes sí (ley de la gravedad); Ias teorías no (origen del sistema solar). ( $3 r$ curso)

Este áltimo alumno considera la ley de la gravedad una verdad estática, aunque reconozca que las teorías sobre el origen del sistema solar tienen carácter temporal.

La idea que revelan las respuestas de esta categoria (que la ley tiene un estatus epistemológico más estructurante que la teoría) se ve aItamente reforzada en las respuestas à la siguiente cuestión.

\section{Estatus epistemológico de las teorías y leyes científicas}

La idea que cada profesor tiene sobre «teorías y leyes científicas» puede ayudar a revelar cuál es su comprensión acerca de epistemología, objetivos y métodos de la ciencia, la cual podrá a su vez influir en el proceso de enseñanza-aprendizaje. De ahí que sea importante esclarecer cuáles son las nociones que los futuros profesores poseen sobre las teorías y leyes científicas.

Una teoría científica debe ser entendida como una uestructura de relaciones lógicas» que interpreta temporalmente un conjunto de fenómenos de un dominio dado de la ciencia. Es un «marco teórico» creado por la mente humana, validado y aceptado por la comunidad científica en un momento dado de la historia de la ciencia y que estructura las leyes cientificas. Según Schwab (1964), cualquier investigación se apoya en la existencia de un marco teórico. Dicho marco es el que capacita a los científicos, cuando encuentran una discrepancia en sus datos, para reconocerla como tal: lo que se observa no «encaja» en la teoría.

Normalmente una teoría científica (puesto que interpreta un dominio particular de la ciencia) engloba una o más leyes científicas, entendidas como las relaciones entre las diferentes magnitudes implicadas en un fenómeno determinado. La teoría científica tiene, por tanto, un estatus más inclusivo que la ley.

En la tabla IV se muestran las frecuencias para las categorías de respuestas a la cuestión:

¿En tu opinión existe alguna diferencia entre una teoría y una ley científica? Si es así, ¿cuál? Da ejemplos que clarifiquen tu respuesta.

La visión antes descrita sobre la construcción del edificio científico no es compartida por gran parte de los futuros profesores, como se puede verificar en la tabla IV.

Tabla IV

Concepciones dominantes entre los futuros profesores sobre estatus de las teorías y leyes científicas.

\begin{tabular}{|l|c|c|}
\hline Categoría de respuesta & $\begin{array}{c}\text { 1r. curso } \\
\%(\mathbf{N}=90)\end{array}$ & $\begin{array}{c}\text { 3r. curso } \\
\%(\mathbf{N}=70)\end{array}$ \\
\hline $\begin{array}{l}\text { Ley con estatus epistemológico } \\
\text { más estructurante que tcoría }\end{array}$ & 80,0 & 43,2 \\
\hline $\begin{array}{l}\text { Ley con estatus epistemológico } \\
\text { menos estructurante que teoría }\end{array}$ & 5,8 & 33,6 \\
\hline Otras & 7,1 & 11,6 \\
\hline No contesta & 7,1 & 11,6 \\
\hline
\end{tabular}


Aunque parece haber un progreso cuando se comparan los resultados de $1^{\circ}(5,8 \%$ de respuestas apropiadas) con los de $3^{\circ}(33,6 \%$ de respuestas apropiadas $)$, es preocupante comprobar que un porcentaje tan grande $(43,2 \%)$ de futuros profesores (en periodo de prácticas) aún presente ideas tan poco científicas sobre la construcción de la ciencia, identificando teoría con idea, opinión, cualquier cosa todavía no sometida a examen y que sólo después de haberlo sido pasa a ser ley. Ejemplos de respuestas que evidencian esta idea son:

En mi opinión existe diferencia entre teoría y ley científica, pucsto que una teoría no pasa de ser algo que se dice con relación a una determinada materia, mientras que la ley científica se asienta sobre bases concretas inamovibles [...] La ley científica rige principios absolutos como la ley de la gravedad. (It. curso)

[...] la teoría científica es generalmente algo descubierto por un científico, una visión individual acerca de un determinado tema, que puede ser o no aceptado por otros científicos; mientras que ley científica es algoestudiado por varios científicos y comprobado experimentalmente, de la cual no existen dudas por lo menos mientras no sean descubiertos otros conocimientos acerca de tal asunto. (3r. curso)

Una teoria cientifica es un conjunto de ideas, de conjeturas no formalizadas totalmente, mientras que una ley es algo concreto, aceptado por todos, es como una «oficialización». (3r. curso)

Es preocupante comprobar que cerca de dos tercios de los futuros profesores (ya con formación cientifica formal finalizada) aún no tienen posicionamientos científicos respecto a la estructura de la ciencia, ni reconocen el papel de las leyes y teorías científicas en su construcción. Para ćstos la ciencia tiene un carácter estático al ser considerada en términos de leyes científicas, pero tiene un carácter dinámico si se considera en términos de teorías. Esto revela que desconocen no sólo qué son las teorías científicas sino también su papel en la construcción de la ciencia y el carácter dinámico de Ia misma.

\section{Relación ciencia-sociedad}

I a opinión pública acerca de la interacción entre ciencia y sociedad es construida en gran medida a través de los medios de comunicación, que en general cnfatizan los aspectos negativos de los resultados de tal interacción (desastres causados por centrales nucleares, efectos de la contaminación, bornba atómica, problemas éticos causados por los nuevos avances de la genética molecular, etc.). Se podría pensar que los individuos encuestados tendrían una visión negativa relativa a este aspecto, y no una visión mixta, deseable, en la cual se valorasen los aspectos positivos. En realidad y a pesar de la existencia de aspectos negativos resultantes del desarrollo de la ciencia, el gran progreso de la humanidad se debe al crecimiento que la ciencia ha experimentado en las últimas décadas.

En la tabla V se muestran las frecuencias correspondientes a las respuestas de la cuestión:
¿Qué consecuencias crees que tienen los resultados de la ciencia en la socicdad?

Tubla V

Concepciones dominantes entre los futuros profesores sobre la relación ciencia-sociedad.

\begin{tabular}{|l|c|c|}
\hline Categoria de respuesta & $\begin{array}{c}\text { Ir. curso } \\
\%(\mathrm{~N}=90)\end{array}$ & $\begin{array}{c}\text { 3r. curso } \\
\%(\mathrm{~N}=70)\end{array}$ \\
\hline Visión optimista & 38,6 & 35,8 \\
\hline Visión pesimista & 0,0 & 2,1 \\
\hline Visión mixta & 52,8 & 48,4 \\
\hline No contesta & 8,6 & 13,7 \\
\hline
\end{tabular}

Los resultados muestran que la interacción cienciasociedad es mayoritariamente entendida como positiva o mixta. Tampoco hay diferencias apreciables entre tos resultados obtenidos con los alumnos de $1^{\circ}$ o de $3^{\circ}$. Una respuesta típica de esta última categoría es:

Los resultados de la ciencia pueden ser varios en las sociedades. Pueden fayorecer en muchos casos a las sociedades con sus innovaciones, ayudando a rebasar ciertos obstáculos o aprovechando algo que nos pueda faverecer. Pueden ocasionar discordias entre sociedades como, por ejemplo, la bomba atómica, que científicamente fue un gran descubrimiento pero socialmente ha traído pánico, miedo y recelo. Y para muchoss causa confusión, principalmente para los menos cultos y los más viejos, que tienen dificultad en aceptar las innovaciones de la ciencia. ( 3 r. curso)

Este resultado está de acuerdo con el estudio de lijnse (1983), el cual concluye que se debe ser escéptico respecto al uso de los medios de comunicación en la construcción de la imagen de la ciencia.

\section{CONCLUSIONES}

Se pueden extraer algunas conclusiones de los resultados de este estudio. Después de algunos años de enseñanza formal de ciencias, los futuros profesores poseen una comprensión muy alejada de la actualmente defendida por los filósofos contemporáneos respecto a la naturaleza de la ciencia -sus objetivos, los procesos seguidos por los científicos en su construcción, la naturaleza del conocimiento y su papel en la sociedad. Desde una perspectiva constructivista creemos, tal como defienden varios autores (Dillon et al., 1994), que la visión de cada futuro profesor sobre los objetivos, procesos, construcción del conocimiento científico e implicaciones de la ciencia en la sociedad influirá el modo en que enseñará cienciá. 
Respecto a la visión de los alumnos sobre el objetivo del trabajo científico, la mayoría consideró que tal objetivo es la búsqueda del conocimiento independientemente de su aplicación. Este resultado permite inferir que la enseñanza a la que probablemente hayan estado sujetos a lo largo de su escolarización no les permitió apreciar el papel que la ciencia fundamental debe tener en el complejo mundo de la ciencia y la tecnología y en la solución de problemas sociales (Lijnse, 1983). En realidad, si se pretende que la educación en ciencia ayude a promover una imagen actual de ésta, se tendrá que enfatizar el importante papel que Ia ciencia fundamental tiene en el mundo científico-técnico. Esto implica, ya sea en la formación inicial de profesores, ya sea en la formación continua, que, a la par del estudio de las teorías, leyes y hechos, se debería prestar mayor atención al papel socializante de la ciencia.

El hecho de que una gran mayoría de estos futuros profesores presenten una visión empírico-positivista de la ciencia hace prever que fácilmente adoptarán estrategias de enseñanza con características de «transmisión cultural» en contradicción con las estrategias innovadoras que permitirían a sus alumnos un aprendizaje más constructivista, defendido por muchos educadores en base a teorías psicológicas actuales (Kelly, 1955).

Parece importante resaltar que las ideas evidenciadas por estos futuros profesores presentan alguna incoherencia. Por ejemplo, respecto a la naturaleza del conocimiento científico, la visión dinámica fue la adoptada por más individuos en cualquiera de los grupos. Sin embargo, respecto al estatus de las leyes y teorías científicas, el $80,0 \%$ de los alumnos de $1^{\circ}$ presentan una visión estática acerca de la ley científica, considerándola mucho más inclusiva que la teoría científica. Esta situación sugiere que la enseñanza de ciencias que hayan recibido, no sólo, pero principalmente en la enseñanza primaria y secundaria, habrá sido de modo compartimentado, lo cual no permitió una visión holística de los diferentes aspectos de la cienciá. Ésta es una idea a explorar en futuras investigaciones. La contribución de la formación específica en este aspecto parece haber sido en cierto modo positiva (el $33,6 \%$ presenta posiciones más próximas a la defendida por los filósofos contemporáncos).

El análisis de los datos relativos a los alumnos de $1^{\circ}$ permite inferir que el modo en que sus profesores les enseñaron ciencias no introdujo concepciones consonantes con el entendimiento sobre su naturaleza a la luz de la nueva filosofía de la ciencia. Esto podría significar que, o bien las estrategias de enseñanza utilizadas no facilitaron una construcción de ideas más científicas en cuanto a la propia naturaleza de la ciencia, o bien que incluso los propios formadores no posefan tales concepciones.

Más preocupantes aún son los datos relativos a los alumnos de $3^{\circ}$, pues de ellos se pueden sacar las mismas conclusiones, pero ahora con repercusiones más graves respecto a la manera en que se les proporcionó formación especifica para la enseñanza de la ciencia. Los resultados de este trabajo permiten concluir que la formación específica inicial, a pesar de no haber sido analizada directamente, no influyó de forma apreciable en las concepciones que los alumnos tienen sobre la naturaleza de la ciencia. De ahí que las implicaciones de este estudio puedan alertar para la introducción de profundas alteraciones en la formación específica inicial de los profesores. Estas alteraciones pasan, por ejemplo, por una revisión de los currículos de la formación espećfica inicial, con el fin de crear espacios para una profunda reflexión sobre la naturaleza de la ciencia, la filosofía e historia de la ciencia y sobre las implicaciones sociales del desarrollo científico. En los comentarios finales pedidos en el cuestionario, varios encuestados señalaban el interés que les había proporcionado responder al cuestionario, pues por primera vez habían reflexionado sobre sus ideas acerca de la ciencia y del conocimiento científico.

Otra alteración que puede ser introducida en la formación específica inicial es la de proporcionar a los futuros profesores la posibilidad de hacer ciencia, integrándolos en equipos de investigación que trabajen en universidades o institutos de investigación. Esto les permitiría comprender mejor los métodos utilizados en la construcción del conocimiento científico.

Parece también imperativo la creación de esbozos de formación continua en los que los profesores, jóvenes y menos jóvenes, sean invitados a reflexionar sobre las mismas cuestiones, integrando ahora su propia experiencia en situaciones de enseñanza-aprendizaje.

Ya se ha comenzado a hacer alguna cosa en Portugal en cuanto a posgrado («masters» y doctorados) con la introducción de espacios destinados a la reflexión sobre estos temas. Sin embargo la situación reclama una intervención más prolongada, lo que requiere antes de nada la preparación de formadores capaces de hacer esta intervención y la sensibilización de las escuelas de formación respecto a la importancia de ayudar a los futuros profesores de ciencia a experimentar la tarea de construir ciencia, «de aprender lo que es ciencia, haciendo ciencia». Éste seráa un terna a abordar en futuros estudios.

\section{NOTAS}

Este estudio ha sido subvencionado por la Junta Nacional de Investigaçăo Científica e Tecnológica (JNICT).

* Este artículo ha sido traducido del portugués por Víctor $\mathrm{M}$. Álvarez. 


\section{REFERENCIAS BIBI,IOGRÁFICAS}

AGLIRRE, J. M, HAGGERTY, S. M. y LINDER, C. J. (1990). Sudent-teachers'conceptions of science, teaching and learning: a case study in preservice science education. Intemational Joumal of Science Education, 12(4), pp. 381-390.

ANDERSON, H.O., HARLY, H. y SAMUEL, K.V. (1986). Nature of Science, 1969 and 1984 ; perspectives of preservice secondary science teachers. School Science and Mathematics, 86, pp. 43-50.

BL,OOM, J.W. (1989). Preservice elementary teachers" conceptions of science: science, theories and evolution. International Journal of Science Education, I1, pp. 401415 .

CACHAPUZ, F. y PRAIA, J. (1992). An Analysis of the conceptions about the nature of scientific knowledge of Portuguese Secondary Science Teachers. Paper presented at the Annual Meeting of the National Association for Research in Science Teaching (NARST), Boston.

CI.EMINSON, A. (1990). Establishing an epistemological base for science teaching in the light of contemporary notions of the nature of science and how children learn science. Journal of Research in Science Teaching, 27, pp. 429-445.

DRIVER, R. y BELL, B. (1985). Students Thinking and the Learning of Science: a constructivist view, School Science Review, 67, pp. 445-456.

DRIVER, R. y OIDHAM, V. (1986). A Constructivist approach to Curriculum Development in Science. Studies in Science Education, 13, pp. 105-122.

DUSCHL, R. A. (1985). Science Education and Philosophy of Science: Twenty-five years of mutually exclusive development. School Science and Mathematics, 85, pp. 541-555.

DUSCHL, R, A. y WRIGHT, E. (I989). A case study of high school tcachers' decision-making models for planning and teaching science. Journal of Research in Science Teaching, 26, pp. 467-502.

ERICKSON, G. L. (1979). Children's conceptions of heat and temperature. Science Education, 63 (2), pp. 221-230.

FORMAN, G. E. (1992). Constructing constructivism. Keynote address given at the anntal meeting of the Association for Constructivist Teaching. San Francisco, CA., October.

FOSNOT,C.T. (1993). Rethinking Science Education: A Defense of Piagetian Constructivism. Journal of Research in Science Teaching, 30, pp. 1189-1201.

GIERE, R. N. (1989). A natureza da ciência numa perspectiva iluminista pós-moderna. Colóquio Ciências, 6, pp. 72-84.

GIL PÉREZ, D. (1992). Contribución de la historia y filosofía de las ciencias a la transformacion de la enseñanza de las ciencias. Conferencia presentada en la Intemational Conference on History of the Physical-Mathematical Sciences and the Teaching of Science. Madrid.

GILBERT, S. W. (1991). Model building and a definition of science. Joumal of Research in Science'Teaching, 28, pp. 73-79.

HARLEN, W. (1987) Teaching and Leaming Primary Science (2a, ed.). England: Teachers College Press.

JOHNSTONE, K. (1987). CIIS in the classroom: constructivist approaches to teaching. Education in Science, 124, pp. 29-30.

KEL,LY, G. (1955) The Psychology of Personal Constructs (Vol. 1 y 2). W. W. Norton and Co. Inc.: Nueva York.

LAKIN, S. Y WELLINGTON, J. (1994). Who will teach the "nature of science»?: teachers'vicws of science and their implications for science education. International Joumal of Science Education, 16, pp. 175-190.

LANT7, O. y KASS, H. (1987). Chemistry'Teachers' Functional Paradigms. Science Education, 7I, pp. 117-134.

LERNER, L.S. y BENNETTA, W. J. (1988). The treatment of theory in textbooks. Science Teacher, 55, pp. 33-41.

OGUNNIYI, M.B. (1982). An analysis of prospective science teachers'understanding of the nature of science. Journal of Research in Science Teaching, 19, pp. 25-32.

POPE, M. y GILBERT, J. (1983). Constructive Science Education. Paper presented at Personal Construct Psychology Conference. Boston.

RITCHIF, S.M. y COOK, J. (1994). Metaphor as a tool for constructivist science teaching. International Joumal of Science Education,16, pp. 293-303.

SALMON, P. (1988). Psychology for Teathers. An Alternative Approach. I.ondres: Hutchinson.

SCHAWB, J.J. (1964). The structure of the natural Sciences, en Ford y Pregno (eds.), The Struture of knowvledge and the Curriculum, pp. 31-49. Chicago: Rand Macnally.

SHEPARDSON, D. y MOJE, E. (1994). The Impact of a Science Demonstration on Children's Understanding of Air Pressure, Journal of Research in Science Teaching, 31, pp. 243-258.

WHITE,F.C.(1989). Knowledge and relativism III: The Sciences. Educ. Phil. and Theory, 15, pp. 1-29.

YAGER, R.E. (1984). The major crisis in science edzcation. School Science and Mathematics, 84, pp. 189-198.

[Artículo recibido en septiembre de 1994 y aceptado en mayo de 1996.] 